Human and Animal Health

Vol.59: e16160109, January-December 2016 http://dx.doi.org/10.1590/1678-4324-2016160109 ISSN 1678-4324 Online Edition

BRAZILIAN ARCHIVES OF BIOLOGY AND TECHNOLOGY

AN INTERNATIONAL JOURNAL

\title{
Insilico Analysis of Phytoconstituents from Allium sativum as Potential Inhibitors of Inha in Mycobacterium tuberculosis
}

\author{
Rmaraj Kannan ${ }^{3}$; Naiyf S. Alharbi²; Shine Kadaikunnan²; Shyam Kumar Rajaram²; \\ Ronaldo Anuf Alexander ${ }^{*}$. \\ ${ }^{I}$ Kamaraj College of Engineering and Technology, Department of Biotechnlogy, Tamilnadu, India; ${ }^{2}$ Department of \\ Botany and Microbiology, College of Science, King Saud University, Riyadh-11451, Saudi Arabia; ${ }^{3}$ Anna \\ University-K B Chandrasekhar Research Centre, Bioinformatics Chennai, Tamil Nadu, India.
}

\begin{abstract}
Tuberculosis is leading cause of death among the global bacterial infections. The main causative for tuberculosis is Mycobacterium tuberculosis, which will survive in its host human being for decades in latent or chronic levels. In addition, the late multidrug resistance at a disturbing rate accompanies the appearance of tuberculosis. The quick spread of resistance to initial stage treatment medications has redirected the focus of the medical community in the creation of an array of new drug against Mycobacterium tuberculosis. The InhA protein is a component of Fatty acid synthetase (FAS) II and exhibits an NADH reliant enoyl-ACP reductase activity. InhA is a vital enzyme of M.tuberculosis in control of cell wall synthesis, which can turn out to be a great focus for the synthesis of antitubercular treatment. Inspired from the offering biological actions of phytoconstituents from Allium sativum, the current research concentrates on looking at novel lead compounds from the plant. Molecular docking studies were carried out employing specific phytoconstituents from A.sativum with the protein InhA target. Ajoene shows much more encouragingresults with a Mol Dock rating of $80.6047 \mathrm{Kcal} / \mathrm{mol}$, as opposed to the typical initial line drug isoniazid (Moldock score: $-58.7028 \mathrm{Kcal} / \mathrm{mol}$ ). Molecular docking prediction indicate that Ajoene could be formulated into a possible treatment drug for Mycobacterium tuberculosis.
\end{abstract}

Key words: Tuberculosis, InhA, Enoyl ACP reductase, Allium sativum, Moldock

\footnotetext{
${ }^{1}$ Authors for correspondence: ronaldoanuf@gmail.com
} 


\section{INTRODUCTION}

Tuberculosis is one of the major global health care issues, caused by the infection of Mycobacterium tuberculosis. M.tuberculosis has about 4111 genes in the circular genome of size $4.4 \mathrm{Mb}$ and $65 \%$ of GC content (Schürch AC, et al., 2010). Almost One third of the human population was being affected by M.tuberculosis of which a major population is from developing countries (Lönnroth K, et al., 2010).

Due to inadequate administration and prolonged use of the first-line treatment drugs like isoniazid rifampicin, ethambutol and streptomycin (VanderWerf MJ, et al., 2012)the emergence of Multidrug resistance in M.tuberculosis strains have been observed around the world with high mortality rates among HIV-positive patients (Jacobs RF, 1994). Improved MDR scenario in M.tuberculosis is also emerging in HIV negative populations (vanRie A, et al., 1999). According to WHO 3.7\% of the new cases of the previously treated patients have been infected with the multidrug-resistant (MDR) M.tuberculosis The reason behind this drug resistance is the primary transmission which is a growing concern in recent days (Zhao $\mathrm{Y}$, et al., 2012). The above mentioned serious concerns has leads to the development of new anti-tubercular drugs.

The internal resistance of M.tuberculosis is due to several factors such as active drug efflux mechanism, highly permeable mycolic acid containing cell wall and the production of drug modifying and drug inactivating enzymes (Ramaswamy S, et al., 1998). M.tuberculosis contains unique signature fatty acids and mycolic acids that are unusually long chain $\alpha$-alkyl and $\beta$ hydroxy fatty acids of 60-90 carbons (Takayama K, et al., 2005). Mycolic acids are the central constituents of the mycobacterial cell wall and the biosynthesis of mycolic acid involves several successive enzymatic reactions involving two enzyme systems FAS-I (Fatty acid Synthase - I) and FAS-II (Fatty acid Synthase - II). In the present study InhA protein a part of Fatty acid Synthase system was selected as a novel drug target.

Plant derived phytochemicals have been traditionally used as natural remedy in treatment of diverse ailments. Usage of medicinal plants demonstrate promising and potential effect in treatment against many human diseases (Ronaldo Anuf, et al., 2014). In this content there is an interest in developing novel lead molecules from plant sources (Ramaraj, et al., 2014).
Garlic (Allium sativum) is a potent medicinal plant widely used as a food item and folk medical treatment for hundreds of years, globally (Rivlin RS, 2001). Garlic is reported to have powerful biological characteristics such antioxidant, antimicrobial, hypoglycemic, anti-cancer, antiinflammatory, immunomodulatory and anticardiovascular effects (Reuter HD, et al., 1996). Various garlic herb extracts have shown highly effective activity against Gram-positive and Gram-negative bacteria such as different strains of Escherichia, Bacillus, Clostridium, Klebsiella, Salmonella, Proteus, Staphylococcus, Streptococcus, Helicobacter pylori (Cellini L, et al., 1996) possibly even acid-fast bacilli (AFB) like MTB (Uchida Y, et al., 1975).

In this study, we aim at exploring novel phytoconstituents from A.sativum as potential lead molecules against the target protein enoyl- ACP reductase (InhA) using Molegro Virtual Docker.

\section{MATERIALS AND METHODS}

\section{Ligand Preparation}

The structure of different phytochemicals from A.sativum were retrieved from Pubchem (https://pubchem.ncbi.nlm.nih.gov/) and Chemspider database (http://www.chemspider.com/). The compounds were converted into compatible format (.sdf) using Open Babel tool. The root mean square gradient value was selected less than $0.001 \mathrm{kcal} / \mathrm{mol}$. The energy minimized structure was used for docking studies.

\section{Protein Preparation}

The three dimensional crystal structure of the protein enoyl- ACP reductase was retained from Protein DataBank (http://www.rcsb.org/pdb/). The bonds, bond orders, explicit hydrogen, charges (calculated by MVD), flexible torsion and Tripos atom types were assigned if they were missing by using 'Protein Preparation' module of Molegro Virtual Docker for the protein enoyl- ACP reductase.

\section{Molecular Docking}

Molgro virtual docking computer program relies upon the innovative hybrid search technique referred to as guided differential evaluation. Docking was carried out applying a grid resolution of $0.30 \AA$ and for all of the 5 independent runs; the highest possible amount of 1,500 iterations were 
carried out on a single population of 50 individuals. The active binding location was regarded as a rigid molecule, however the ligands were regarded to be flexible, i.e. any non-ring torsions were permitted. Default configurations were employed for every calculation.
Phyto-constituents from A.sativum (Table 1.) were docked with enoyl- ACP reductase using Moldock module in the Docking wizard of MolDock Virtual docking software. Moldock uses the Scoring function derived from Piecewise Linear Potential (PLP) scoring function.

Table 1. Selected phyto-compounds of A.sativum

S.No Compound Name Compound ID $\quad$ Structure

\section{(CID)}

1. Ajoene

2. Allicin

3. Alliin

5386591

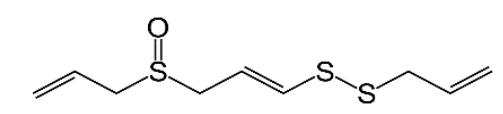

4. Allyl sulfide

5. Ascorbic acid

54670067

6. Cycloalliin

(Chemspider ID)

8. Diallyl Disulfide

9. Diallyl Trisulfide

10. Galacturonic acid

441476

11. Methylallyl

trisulfide

12. S-allylcysteine
87310

65036

11617

193294

11110

16590

16315

61926
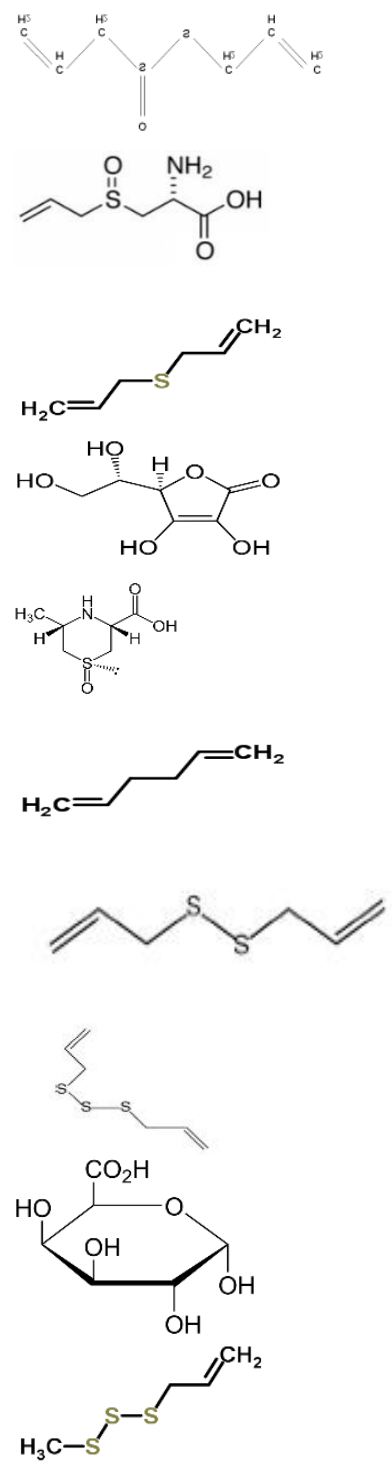

98280

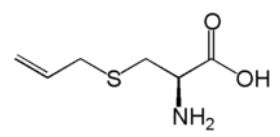




13. Scorodonin 189818

\section{RESULTS AND DISCUSSIONS}

Natural products and some of their derivatives have shown promising activity against M.tuberclosis highlighting their potential as a powerful source of new class of drugs. A number of genetic mutation were seen to have taken place generally between codons 138 and 328. The Ser315Thr mutation is most frequent and present in more than $40 \%$ of every INH resistant strains. These strains needs to be targeted with potent drug molecues to attain the best possible medicinal effects. Therefore, In the present study library of natural compounds from A.sativum has been screened against enoyl- ACP reductase and concluded our results using average binding affinity of these compounds against them. The selected phytocompounds from A.sativum has shown potent binding affinity against enoyl- ACP reductase.

\section{Prediction of Binding site}

Detection of cavities is the more important step in the protein-ligand interaction. The cavities present in the protein enoyl- ACP reductase is detected based on the cavity detection algorithm in MVD tool (Thomsen R, et al., 2006). The volume of the cavities is listed in Table 2. In most cases, the cavities with the largest size and volume is associated with the binding site. Cavity 2 and Cavity 3 have the larger volume of $928.256 \AA^{3}$ "(Fig. 1 A)" and 501.248 $\AA^{3}$ "(Fig. 1 B)" respectively. The cavity with larger size has been selected as the binding site for the protein enoylACP reductase during docking with Molgro Virtual Docker "(Fig. 2)".

Table 2. Volume of cavities

\begin{tabular}{llc}
\hline S.No & Cavities & Volume of cavities $\left(\AA^{\mathbf{3}}\right)$ \\
\hline 1. & Cavity 1 & 416.256 \\
2. & Cavity 2 & 928.256 \\
3. & Cavity 3 & 501.248 \\
4. & Cavity 4 & 428.544 \\
$\mathbf{5 .}$ & Cavity 5 & 418.204 \\
\hline
\end{tabular}

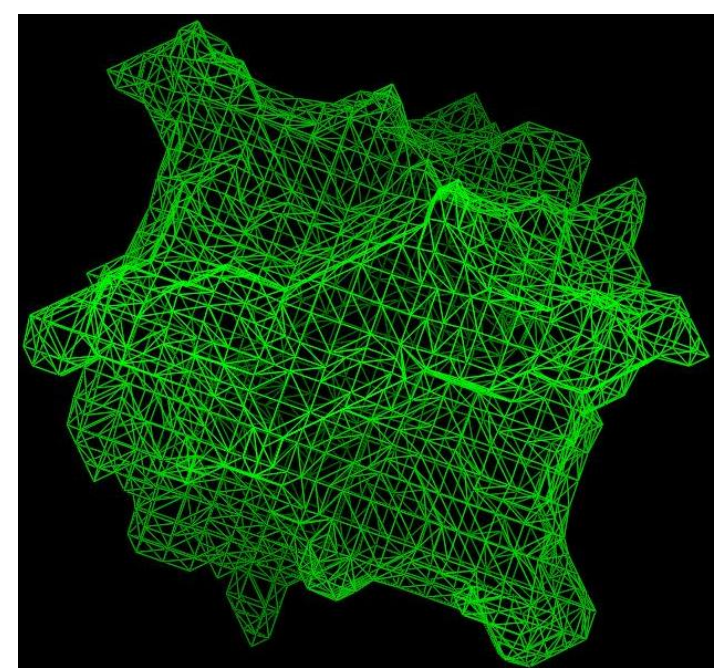

Fig. 1 A Binding site for enoyl- ACP reductase cavity-1

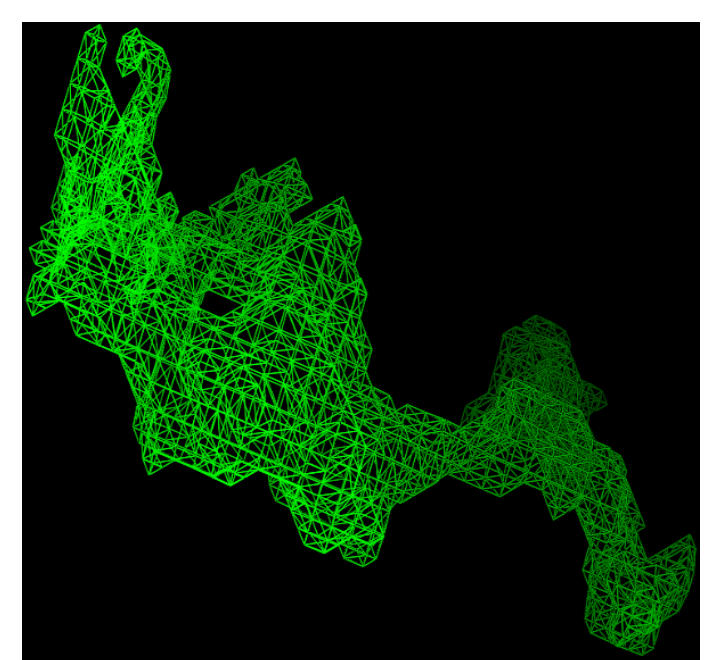

Fig. 1 B Binding site for enoyl- ACP reductase cavity -2 


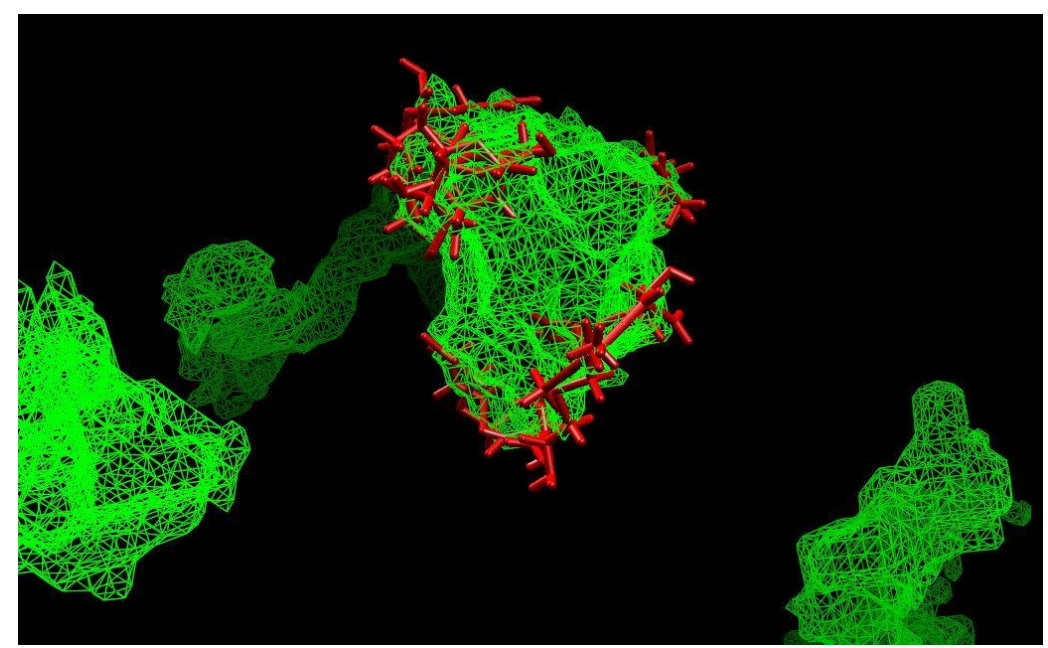

Fig. 2 Selected ligands bind with cavity -1 predicted by MVD tool

\section{Molecular Docking}

In recent scenario, computational methods such as molecular docking tools are used to design novel lead molecules by exploring the interaction between the protein and the ligand molecules (Srivastava, et al., 2010). The docking analysis of the selected phyto-compounds from A.sativum with the protein enoyl- ACP reductase was performed using MVD. The inhibition susceptibility was determined using moldock score which was generated by MVD.

The receptor bound ligand was docked deeply in binding pocket region by strong interaction. The active compound Ajoene binds with the receptor with a moldock score of -80.6047 (Table 3 ). It binds to the Ile -257 amino acid in the cavity 2 (Fig. $3 \mathrm{~A}$ ) and has only one Hydrogen bond interaction. Allin expressed a hydrogen bond score of -9.707
$\mathrm{Kcal} / \mathrm{mol}$ and with four amino acid interactions at different domains of the target protein "(Fig. 3 B)". Ascorbic acid had a moldock score of -71.4068 $\mathrm{Kcal} / \mathrm{mol}$ and the hydrogen bond interactions were high as compared to that of Ajoene. Compounds such as methylallyl trisulfide and diallyl shows very less Moldock score and hence have a very less affinity for the target.

The H-Bond score for Ascorbic acid was -14.5056 Kcal/mol. There are 9 amino acid residues from different domains of enoyl- ACP reductase involved in interaction with Ascorbic acid "(Fig. 3 C)". Scorodonin had four hydrogen bond interaction with 2 amino acid residues "(Fig. 3 D)". Table 4. indicated that the amino acids, Ile 257, Ser 152, and Arg 173 are found common in most compounds.

Table 3. Docking energies of A.sativum for enoyl- ACP reductase

\begin{tabular}{|c|c|c|c|c|c|}
\hline S.No & Compound Name & $\begin{array}{l}\text { Moldock } \\
\text { Score } \\
\text { (Kcal/mol) }\end{array}$ & $\begin{array}{l}\text { Rerank } \\
\text { score } \\
\text { (Kcal/mol) }\end{array}$ & $\begin{array}{l}\text { HBond } \\
\text { (Kcal/mol) }\end{array}$ & $\begin{array}{l}\text { Docking score } \\
\text { (Kcal/mol) }\end{array}$ \\
\hline 1. & Ajoene & -80.6047 & -67.878 & -0.114737 & -80.048 \\
\hline 2. & Scorodonin & -74.033 & -58.872 & -4.62487 & -76.96 \\
\hline 3. & Alliin & -72.1619 & -61.8725 & -9.70757 & -77.939 \\
\hline 4. & Ascorbic acid & -71.4068 & -63.597 & -14.5056 & -85.060 \\
\hline 5. & Thiosulfinate & -69.7631 & -56.3802 & -4.83278 & -69.009 \\
\hline 6. & Cycloalliin & -67.5228 & -56.5924 & -5.39142 & -74.059 \\
\hline 7. & Allicin & -66.6876 & -52.3595 & -1.72242 & -65.644 \\
\hline 8. & S-allylcysteine & -63.5625 & -37.6933 & -9.19796 & -68.176 \\
\hline 9. & Diallyl trisulfide & -62.0729 & -46.8931 & 0 & -61.558 \\
\hline 10. & Diallyl disulfide & -60.7435 & -51.4489 & 0 & -59.583 \\
\hline 11. & Galacturonic acid & -54.8406 & -56.9334 & -16.1429 & -69.654 \\
\hline 12. & Allyl sulfide & -51.9668 & -43.8319 & 0 & -51.724 \\
\hline 13. & Methylallyl trisulfide & -49.9732 & -40.1364 & 0 & -49.356 \\
\hline 14. & Diallyl & -49.4581 & -40.5511 & 0 & -48.913 \\
\hline
\end{tabular}




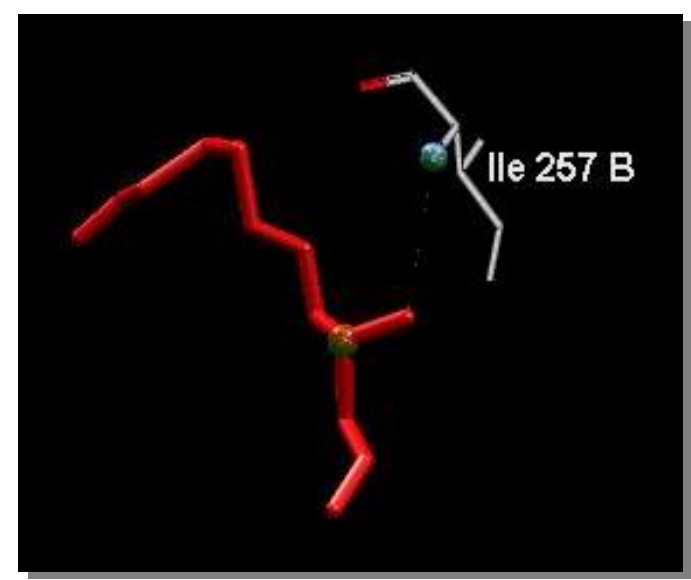

Fig. 3 A Interaction of Ajoene $\mathrm{H}_{2}$ bond

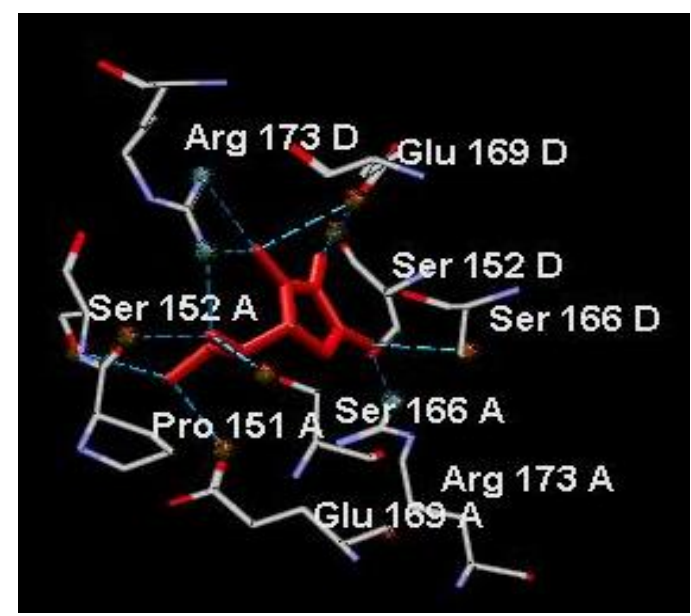

Fig. $3 \mathrm{C}$ Interaction of Ascorbic acid $\mathrm{H}_{2}$ bond

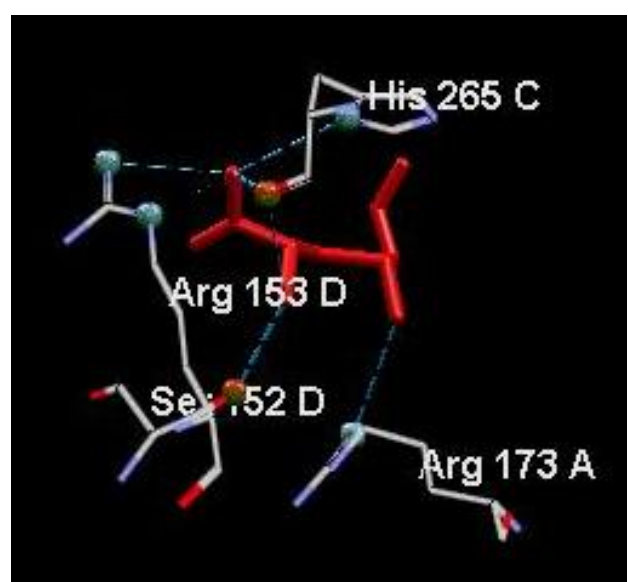

Fig. 3 B Interaction of Allin $\mathrm{H}_{2}$ bond

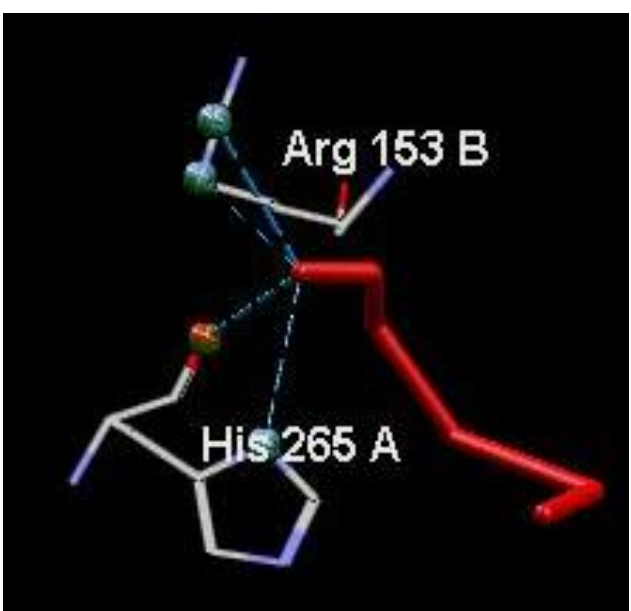

Fig. 3 D Interaction of Scordinin $\mathrm{H}_{2}$ bond

Table 4. Amino acid residue of enoyl- ACP reductase involved in binding with phytoconstituents from A.sativum

\begin{tabular}{|c|c|c|c|}
\hline S.No & Compound Name & $\mathrm{H}_{2}$-interaction with Amino acid & No. of $\mathrm{H}_{2}$ bond \\
\hline 1. & Ajoene & Ile 257 & 1 \\
\hline 2. & Scorodonin & Arg 153 , His 256 & 4 \\
\hline 3. & Alliin & Ser 152, Arg 153, Arg 173, His 265 & 7 \\
\hline 4. & Ascorbic acid & Pro 151, Ser 152, Ser 166, Glu 169, Arg 173 & 12 \\
\hline 5. & Thiosulfinate & Arg 173 , Gly 255 & 2 \\
\hline 6. & Cycloalliin & Ser 152 , Arg 173, Gly 255, Asp 256, Ile 257 & 5 \\
\hline 7. & Allicin & Arg 173 & 1 \\
\hline 8. & S-allylcysteine & Asp 256, Ile 257, Tyr 259 & 7 \\
\hline 9. & Diallyl trisulfide & -- & -- \\
\hline 10. & Diallyl disulfide & -- & -- \\
\hline 11. & Galacturonic acid & Ser 166, Glu 169, Ser 170, Arg 173, & 12 \\
\hline 12. & Allyl sulfide & -- & -- \\
\hline 13. & Methylallyl trisulfide & -- & -- \\
\hline 14. & Diallyl & -- & -- \\
\hline
\end{tabular}

The rate limiting step and final step Mycobacterium Enoyl-ACP reductase catalyzes the final step and rate limiting step in the fatty acid synthesis (mycolic acid) by utilizing $\mathrm{NADH}$ to reduce trans double bond of longer fatty acyl substrates (Moir DT, 2005, Rozwarski DA, et al., 1998) and are validated as the excellent target for drug development against M.tuberculosis (Yamada H, et 
al., 1995). The replacement of an amino acid in the NADH binding site of $\operatorname{Inh} A$ apparently results in INH resistance, preventing the inhibition of mycolic acid biosynthesis (Zhang YM, et al., 2006). Various genetic mutations have been observed to occur usually between codons 138 and 328 (Ramaswamy S, et al., 1998). The Ser-315-Thr mutation is most frequent and found in about $40 \%$ of all INH-resistant strains (Marttila HJ, et al., 1998, Zhang YM, et al., 2006). The mutation leads to the enzyme lacking the ability to activate $\mathrm{INH}$, but preserves $50 \%$ of catalase peroxidase activity. The modified catalase-peroxidase offers high-level resistance to INH (Telenti A, et al., 1993). So the biological activity of INH is reduced due to the mutation in InhA. Therefore, there is an urgent need for novel intervention strategies to target TB.

Ajoene shows the potent inhibitory activity against the InhA protein. Ajoene (IUPAC name: (Z)-1(prop-2-enyldisulfanyl)-3prop-2-enylsulfinylprop1-ene) is the organosulfur compound, which contains sulfoxide and disulfide groups. Ajoene is reported for the anti-cancer activity (Roger, et al., 2008), anti-bacterial activity (Torres, et al., 2012), anti-fungal activity (Ledezma, et al., 2006) and wide possess wide range of biological applications. Development of Ajoene as a potential inhibitor would assist in effective medication for the disease with minimal or less toxic effects.

\section{CONCLUSION}

The present molecular docking studies provide insights into inhibition of InhA protein by phytoconstituents from A.sativum. Docking study suggest that Ajoene has a high binding affinity for InhA protein, which is much higher than the known inhibitor isoniazid. This study has led to the development of novel lead molecules for the treatment of Tuberculosis. Further these lead compounds can be used for designing more effective inhibitors of InhA.

\section{ACKNOWLEDGMENTS:}

The authors extend their sincere appreciations to the Deanship of Scientific Research at King Saud University for its funding this Prolific Research Group (PRG-1437-36).

\section{REFERENCE}

Cellini L, DiCampli E, Masulli M, DiBartolomeo S and Allocati N, Inhibition of Helicobacter pylori by garlic extract (Allium sativum), FEMS Immunol. Med.Micrbiol., 13, 1996, 273-277.

Jacobs RF, Multidrug-resistant tuberculosis, Clinical Infectious Diseases, 19 1994, 1-10.

Ledezma E and Apitz-Castro R, Ajoene the main active compound of garlic (Allium sativum): a new antifungal agent, Revista Iberoamericana de Micología, 23, 2006, 75-80.

Lönnroth K, Castro KG and Chakaya JM, Tuberculosis control and elimination 2010-50: cure, care, and social development, Lancet, 375 2010, 1814-1829.

Marttila HJ, Soini H and Eerola E, A Ser315Thr substitution in $\mathrm{KatG}$ is predominant in genetically heterogeneous multidrug-resistantMycobacterium tuberculosis isolates originating from the St. Petersburg area in Russia, Antimicrob Agents Chemother, 42, 1998, 2443-2445.

Moir DT, Identification of inhibitors of bacterial enoylacyl carrier protein reductase, Infectious Disorders - Drug Targets, 5, 2005, 297-305.

Ramaraj K and Ronaldo Anuf A, Inhibition of FGFR2 signal transduction in Acne Vulgaris using Bioactive Flavonoids: An Insilico approach, Asian Journal of Medical and Pharmaceutical Sciences, 2, 2014, 136142.

Ramaswamy S and Musser JM, Molecular genetic basis of antimicrobial agent resistance inMycobacterium tuberculosis: 1998 update, Tubercle and Lung Disease, 79, 1998, 3-29.

Reuter HD, Koch HP and Lawson LD, Therapeutic effects and applications of garlic and its preparations. In: Garlic. The Science and Therapeutic Application of Allium sativum L. and Related Species, Baltimore: Williams \& Wilkins, 1996, 135-212.

Rivlin RS, Historical perspective on the use of garlic, J. Nutr., 131, 2001, 951-954.

Roger H, Catherine HK, Parker IM, Mino RC, Philip R, Susan T, Francois T and Thozama Q, Substituted ajoenes as novel anti-cancer agents, Bioorganic \& Medicinal Chemistry Letters, 18, 2008, 5277-5279.

Ronaldo Anuf A, Breethi N, Shahila S and Ramaraj K, Insilico Analysis of Plumbagin and its synthetic analogues as potential Bcl-2 Inhibitors, Asian Journal of Medical and Pharmaceutical Sciences, 2, 2014, 108-114.

Rozwarski DA, Grant GA, Barton DHR, JacobsJr WR and Sacchettini JC, Modification of the NADH of the isoniazid target (InhA) from Mycobacterium tuberculosis, Science, 279, 1998, 98-102.

Schürch AC, Kremer K, Kiers A, Daviena O, Boeree MJ, Siezen RJ, Smith NG and vanSoolingen D, The tempo and mode of molecular evolution of Mycobacterium tuberculosis at patient-to-patient scale, Infect Genet Evol, 10 2010, 108:114.

Srivastava V, Gupta SP, Siddiqi MI and Mishra BN, Molecular docking studies on quinazoline antifolate 
derivatives as human thymidylate synthase inhibitors, Bioinformation, 4, 2010, 357-365.

Takayama K, Wang C and Besra GS, Pathway to synthesis and processing of mycolic acids inMycobacterium tuberculosis, Clin. Microbiol. Rev., 18 2005, 81-101.

Telenti A, Imboden P and Marchesi F, Detection of rifampicin-resistance mutations in Mycobacterium tuberculosis, Lancet, 341 1993, 647-650.

Thomsen $\mathrm{R}$ and Christensen MH, MolDock: A new technique for high-accuracy molecular docking., J Med Chem, 49, 2006, 3315-3321.

Torres $\mathrm{J}$ and Romero $\mathrm{H}$, In vitro antifungal activity of ajoene on five clinical isolates of Histoplasma capsulatum var. capsulatum, Revista Iberoamericana de Micología, 29, 2012, 24-28.

Uchida Y, Takahashi T and Sato N, The characteristics of the antibacterial activity of garlic, Jpn. J. Antibiotics, 28, 1975, 638-642.

VanderWerf MJ, Langendam MW, Huitric E and Manissero D, Multidrug resistance after inappropriate tuberculosis treatment: a metaanalysis, Eur Respir J, 39 2012, 1511-1519.
vanRie A, Warren RM and Beyers N, Transmission of a multidrug-resistantMycobacterium tuberculosis strain resembling 'strain W' among noninstitutionalised, human immunodeficiency virusseronegative patients, J. Infect. Dis., 180 1999, 1608:1615.

Yamada H, Shiomi K, Xu Q, Nagai T, Shibata M and Oya L, New glycosidases inhibitors, panosialins D and wD produced by Streptomyces sp. OH-5186, J. Antibiot., 48, 1995, 205-210.

Zhang YM, White SW and Rock CO, Inhibiting bacterial fatty acid synthesis, J. Biol. Chem., 281, 2006, 17541-17544.

Zhao Y, Xu S and Wang L, National survey of drugresistant tuberculosis in China, The New England Journal of Medicine, 366, 2012, 2161-2170.

Received: January 15, 2016; Accepted: April 25, 2016 